
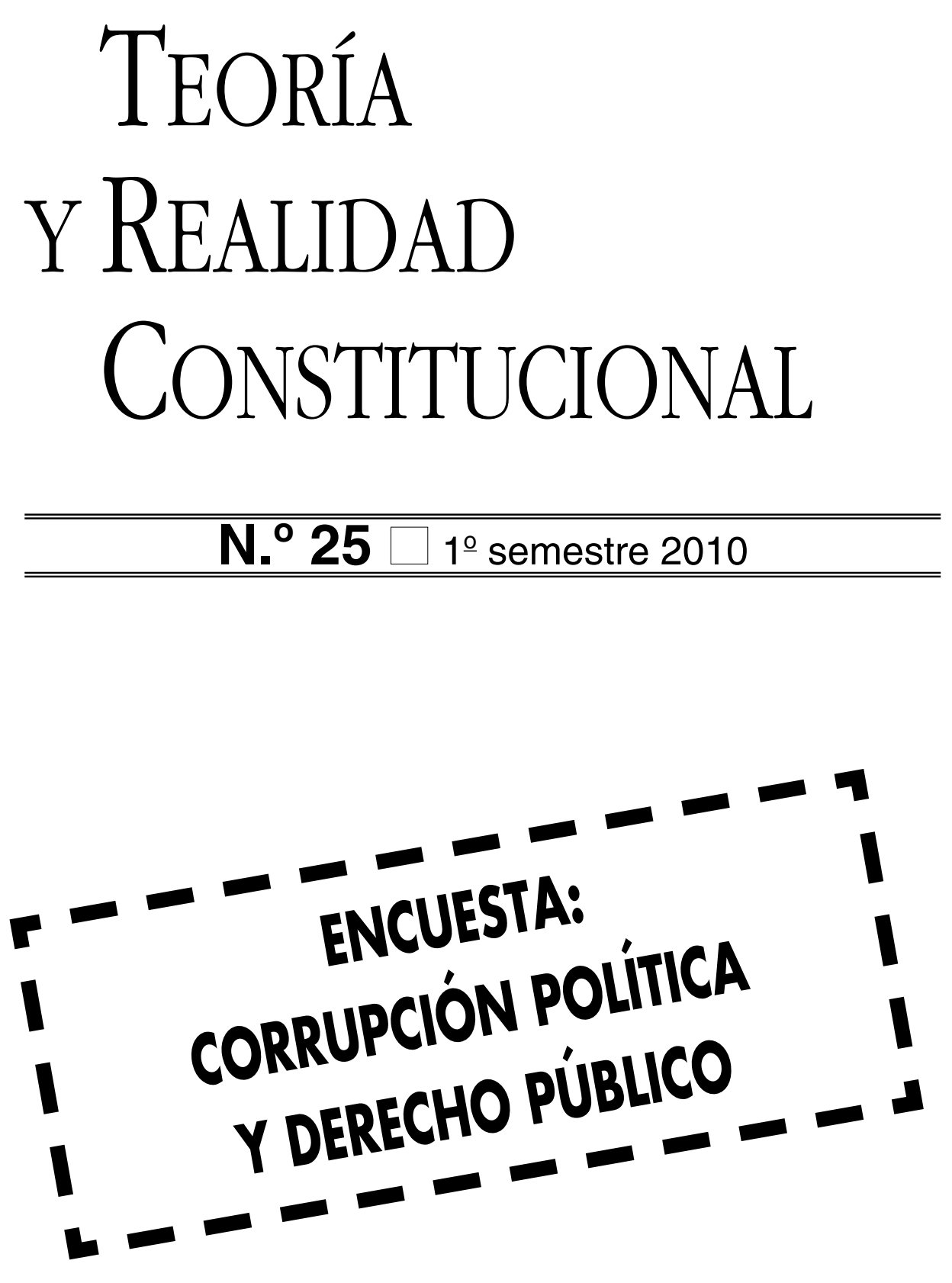


\title{
PROBLEMAS DE LA PROMULGACIÓN DE UNA CONSTITUCIÓN BRITÁNICA*
}

\author{
VERNON BOGDANOR \\ Profesor de Derecho Público \\ Universidad de Oxford \\ STEFAN VOGENAUER \\ Profesor de Derecho Comparado \\ Universidad de Oxford
}
SUMARIO
I. Alcance.
II. El es y el debe ser: el papel de las con- venciones.
III. Elaboración, ratificación y modificación.
IV. Conclusiones

En la obra The Law of the Constitution, Dicey escribió que un autor británico en materia constitucional "tiene buenas razones para envidiar a los profesores de países como Francia...o los Estados Unidos, dotados con constituciones cuyos términos se encuentran en documentos impresos, conocidos por todos los ciudadanos y accesibles a cualquier hombre que sepa leer.. ${ }^{1}$ Gran Bretaña sigue siendo, con Nueva Zelanda e Israel, una de las tres democracias que todavía no tienen una constitución "escrita" o, mejor dicho, "codificada".

De vez en cuando, se apunta que sería mejor que Gran Bretaña contara con una constitución entendida en ese sentido. El partido laborista, durante el largo periodo que permaneció en la oposición entre 1979 y 1997, llegó a la

* Traducción de M. SALVADOR MARTÍNEZ y S. SÁNCHEZ GONZÁLEZ, Profesores Titulares de Derecho Constitucional de la UNED. Trabajo publicado originalmente en la revista Public Law, en el número de primavera de 2008. Los traductores han decidido mantener el sistema de citas en las notas a pie que utilizó el autor en la publicación original del trabajo.

1 A.V. DICEY, Introduction to the Study of the Law of the Constitution, $10^{\mathrm{a}}$ ed. (Macmillan, 1959), pág. 4. 
conclusión de que los pesos y contrapesos constitucionales pueden servir para controlar lo que se ha convertido, a su juicio, en una dictadura electiva, y, en 1991, un panel de expertos simpatizante del partido laborista, el Institute for Public Policy Research, publicó una Constitution for the United Kingdom muy detallada. ${ }^{2}$ Desde entonces la idea ha ganado fuerza, en parte debido al gran número de leyes constitucionalmente materiales que han sido promulgadas desde 1997. Parece como si hubiéramos estados sometidos a un proceso, único en el universo democrático, con la excepción de la experiencia israelí, de transformación de una constitución sin codificar en una codificada, de manera gradual y fragmentariamente, sin ningún tipo de consenso sobre cual sería el resultado final. Sería lógico, por lo tanto, proponer que concluya ya ese proceso con la elaboración de una constitución y su promulgación en un solo texto. En octubre del año 2006, Lord Goldsmith, entonces Fiscal General, invitó a un debate sobre el tema ${ }^{3}$; y Gordon Brown, como Ministro de Finanzas y como Primer Ministro, ha señalado que una constitución codificada podría fortalecer el sentir británico. El Informe publicado poco después del nombramiento de Gordon Brown como Primer Ministro apunta que "hay ahora un reconocimiento creciente de la necesidad de clarificar no solo lo que significa ser británico, sino lo que significa el Reino Unido. Esto podría en breve llevar a un acuerdo entre el ejecutivo y el parlamento sobre una constitución escrita.." ${ }^{4}$ Entre los partidos de la oposición, los liberal-demócratas se han mostrado comprometidos desde hace tiempo con esa idea.

En una carta al periódico The Times, del 8 de febrero del 2006, el Sr. Stephen Hockman, Presidente del Bar Council* para Inglaterra y Gales, dijo que, después de un periodo de reformas constitucionales, "la inmensa mayoría de nosotros carece de un entendimiento claro y comprensivo de cuáles son realmente los términos de nuestra constitución." Y, en consecuencia, propuso adoptar:

"....una medida codificadora, de modo que se contengan en una sola norma todos los principios constitucionales clave y los procedimientos en que basa el gobierno del país. Ese instrumento permitirá que cada ciudadano conozca y comprenda como funciona la Constitución Británica y, sobre todo, proporcionará un marco de referencia claro para juzgar no solo las decisiones y acciones de los que nos gobiernan, sino también cualquier propuesta de reforma que puedan hacer."

2 Institute for Public Policy Research, 1991. Cfr. sobre este tema, J. CORNFORD, "On Writing a Constitution" (1991) 22 Parliamentary Affairs 558-571; D. OLIVER, "Written Constitutions: Principles and Practices" (1992) 45 Parliamentary Affairs 135-152; y R. BRAZIER, "Enacting a Constitution" (1992) 13 Statute Law Review 104-127. Cfr., también, BRAZIER, "How Near is a Written Constitution?" (2001) 52 Northern Ireland legal Quarterly 1-19.

3 En una entrevista en el programa "Sunday Live" en la Sky Televisión, el 8 de octubre de 2006.

4 The Governance of Britain, Cm 7170 (julio 2007), par. 212.

* N. del T.: el Bar Council es la Asociación profesional de los abogados para los ámbitos de Inglaterra y Gales. 
La redacción de una constitución codificada es asimismo, sin duda, materia central en muchos cursos universitarios de derecho constitucional. Uno de los autores de este artículo recuerda haber asistido hace muchos años, como estudiante en Oxford, a un seminario organizado con el propósito de elaborar ese documento, que impartían F. H. Lawson, H. W. R. Wade, y el decano de los estudios constitucionales en Gran Bretaña, K. C. Wheare. ${ }^{5}$

Los autores de este artículo decidimos emprender una experiencia similar en Oxford en el semestre de otoño del 2006. Tuvimos reuniones semanales durante las cuales grupos reducidos de estudiantes y licenciados, de las Facultades de Derecho y de Ciencias Políticas, preparaban para su discusión una sección específica de la constitución, por ejemplo, la relativa al poder legislativo, al judicial o a los derechos humanos. Al final, se elaboró una constitución completa que fue publicada por el Smith Institute, como apéndice a un conjunto de ensayos bajo el título Towards a New Constitutional Settlement - Hacia un Nuevo Acuerdo Constitucional-, editado por el parlamentario Chris Bryant y publicado también en la revista Political Quarterly en el número de 2007. ${ }^{6}$

El experimento puso sobre la mesa una serie de problemas interesantes relativos tanto al deseo como a la posibilidad real de promulgar una constitución codificada. En un artículo reciente hemos discutido con Tarunabh Khaitan la cuestión de "lo deseable" de una constitución británica.7 Lo que pretendemos a continuación es considerar la cuestión de la factibilidad o, mejor dicho, los problemas que hay que resolver si se decide promulgar una constitución de Gran Bretaña. No nos ocuparemos aquí de asuntos de enjundia tales como, por ejemplo, el tratamiento de la idea de la independencia judicial o los derechos que deberían incluirse en la constitución. Aún así, los problemas implicados son amplios y complejos y poco podemos hacer salvo apuntarlos en una forma breve y crudamente.

\section{ALCANCE}

El primer problema, y el más obvio, es decidir que es lo que hay que incluir en el texto constitucional. Stephen Hockman, en la carta a The Times, sugería que deberían incluirse "todos los principios constitucionales y procedimientos clave, en los que se basa el gobierno de nuestro país" (el subrayado es nuestro). Pero quizás esa es una aspiración utópica. Después de todo, una constitución contiene una selección de las normas que controlan la actuación del gobierno en un Estado. Si, además, una constitución debe tener, como

$5 \mathrm{El}$ autor de, entre otras obras, Modern Constitutions (Oxford University Press, publicada por vez primera en 1951). N. del T.: Esta obra fue publicada en español con el título Las constituciones modernas por Editorial Labor, Barcelona, 1971.

6 Smith Institute, 2007.

7 "Should Britain have a Written Constitution", The Political Quarterly, (2007) 78, págs. 499517. 
Stephen Hockman desea, una función educadora, necesitaría ser relativamente breve. Por lo tanto, habría que hacer una selección entre las leyes y, quizás, las convenciones, para recoger aquellas que expresan «los principios y procedimientos constitucionales". ${ }^{8}$

Dicey, que desde luego no concedió valor alguno a la promulgación de una constitución para Gran Bretaña, analizó la Law of the Constitution, mediante la selección de los principios esenciales de la constitución - la soberanía del parlamento, el rule of law y la dependencia de las convenciones jurídicas. En su libro Some Problems of the Constitution, Marshall y Moodie señalaron un cuarto principio, el de la responsabilidad ministerial. La Ley de Reforma Constitucional de 2005 reitera el principio del rule of law y destaca la importancia de la independencia judicial, que podría ser considerado el quinto principio de la constitución.?

Sin embargo, la elaboración de una constitución sobre la base de esos principios conlleva dificultades considerables, puesto que son demasiado generales y podrían entrar en conflicto. A decir verdad, Marshall y Moodie señalaron que buena parte de la historia constitucional de Gran Bretaña en el siglo XX "podría representarse como un conflicto" entre el principio de la responsabilidad ministerial y los principios de "la soberanía del parlamento y el rule of law". ${ }^{10}$ No es fácil en modo alguno determinar el peso relativo otorgable a cada principio.

Por lo tanto, no sería posible resolver el problema de seleccionar aquellas leyes y convenciones que expresan "los principios y procedimientos constitucionales", deduciéndolos de principios constitucionales particulares, puesto que estos principios son necesariamente genéricos en la forma, a veces entran en conflicto, y están sometidos a interpretaciones muy diferentes y quizás controvertidas políticamente. Además, como veremos, si se toma realmente en serio el principio de la soberanía del parlamento, no tiene sentido elaborar y promulgar una constitución.

En Modern Constitutions, Wheare escribe que en un Estado unitario:

"...la Constitución no necesita más que procurar la estructura, en términos generales, de los poderes ejecutivo, legislativo, y judicial; el perfil de la naturaleza de sus relaciones recíprocas; y la naturaleza de las relaciones entre dichos poderes y la comunidad. .11

8 Sobre la distinción entre una constitución "del pueblo" y una constitución de "abogados", véase The Political Quarterly, (2007) 78, págs. 503-505.

9 En Canadá, el Tribunal Supremo ha mantenido en los años recientes que la constitución implica ciertos principios constitucionales no escritos, por ejemplo, la independencia judicial, y ha comenzado a aplicarlos como si hubieran sido formulados de manera expresa. Cfr. P. W. HOGG, Constitutional Law of Canada, $5^{\mathrm{a}}$ ed. (Scarborough, Ontario: Thomson/Carswell, 2006) págs. 15,52 .

10 G. MARSHALL Y G. C. MOODIE, Some Problems of the Constitution, $5^{\mathrm{a}}$ ed. (Hutchinson, 1971) pág. 11.

11 K.C. WHEARE, Modern Constitutions, $2^{\mathrm{a}}$ ed. (Oxford University Press, 1966) pág. 34. (N. del T.: op. cit. en español, pág. 39). 
Además, es cierto que se espera de la mayoría de los documentos modernos que contengan preceptos que definan la nacionalidad y la aplicación territorial de la constitución, junto con los artículos que regulan los distintos niveles territoriales de gobierno y sus competencias respectivas, amén de una declaración o catálogo de derechos. Más allá de este contenido, la diversidad entre las constituciones promulgadas es enorme. Bastantes cuentan con un preámbulo que contiene un "establecimiento de objetivos", pero otras no. Algunas añaden derechos económicos y sociales a la relación habitual de derechos, y otras no lo hacen. Existe, por lo tanto, un núcleo central, pero, al margen del mismo, no hay acuerdo sobre qué debería incluirse en una constitución.

El problema de seleccionar las normas que debería recoger una constitución tiene que plantear dificultades más difíciles de afrontar en Gran Bretaña que en la mayoría de las democracias que han promulgado una constitución. Esto es debido a que "si buscamos los orígenes de las constituciones modernas, veremos que prácticamente sin excepción, fueron creadas y adoptadas porque el pueblo deseaba procurarse una nueva base para el establecimiento de su sistema de gobierno." ${ }^{12}$ Muchos de los constituyentes no tienen, por lo tanto, que afrontar la dificultad de seleccionar entre un legado considerable de leyes existentes, costumbres y convenciones. Los padres constituyentes norteamericanos, por ejemplo, adoptaron una constitución de novo, para sustituir una entidad confederal por una federal. La Constitución de la India de 1950 representó la independencia nacional. Alemania en 1949 y Francia en 1958 aprobaron constituciones nuevas que suponían el comienzo de nuevos regímenes. En general las constituciones se promulgan cuando llega un determinado momento constitucional, después de una guerra, de una revolución, o de la independencia colonial. Gran Bretaña, sin embargo, intentaría adoptar una constitución que comprendiera y resumiera el funcionamiento de un régimen político en plena actividad. Además, cualquier régimen con una constitución sin codificar está necesariamente sujeto a un proceso inacabado de adaptación y cambio. En consecuencia, la promulgación de una constitución equivaldría a la tentativa de aprehender la esencia de una tradición cambiante mientras está siendo descrita.

Sydney Low escribió en 1904 que:

"Existe una razón por la que es tan difícil describir el sistema de gobierno inglés. Cualquier explicación debe ser como la imagen de una persona viva. Si deseas ver exactamente como es el original, no te remites a una fotografía tomada hace veinte o treinta años. Los rasgos pueden ser los mismos, pero su expresión, su presentación y todo su carácter han cambiado. En el intervalo entre un examen de nuestra política pública y otro, quizás no se haya alterado prácticamente el aspecto formal, pero la parte funcional, orgánica y convencional se ha modificado de muchas maneras. Es cierto 
que los elementos estructurales muestran una permanencia superficial maravillosa. La Corona, las dos Cámaras del Parlamento, el Consejo de Ministros (es decir, el Gabinete), el Cuerpo Electoral, el Poder Judicial, y las relaciones mutuas de estos poderes y autoridades son el material de que se sirven todos los historiadores y los juristas. Es la misma maquinaria, o al menos una maquinaria a la que se ha dado una mano de pintura para que parezca la misma."13

En Gran Bretaña el problema para el constituyente es decidir qué elementos de un sistema de gobierno funcionando a pleno rendimiento deberían seleccionarse por su especial significación para ser incluidos en la constitución, al tiempo que el propio sistema está cambiando y, quizás, muy rápidamente; puesto que no hay razón para creer que la era de las reformas constitucionales que se inició en 1997 haya concluido.

Una cuestión que probablemente puede resultar muy controvertida es la de si el papel de los partidos políticos y el sistema electoral deberían especificarse en la constitución. La Constitución norteamericana, anterior a la formación de los partidos políticos organizados, nada dijo al respecto. Los Padres Fundadores propusieron una constitución que reflejaba una concepción de la separación de poderes propia del siglo XVIII. Muchas constituciones posteriores han seguido el ejemplo norteamericano, aunque la existencia de partidos políticos es ahora una condición sine qua non del gobierno democrático. La Constitución de Alemania de 1949 es una de las pocas, sin embargo, que pretende regular las actividades de los partidos. El artículo 21 otorga al Tribunal Constitucional Federal la competencia de declarar inconstitucional cualquier partido que por sus fines o por el comportamiento de sus afiliados "trate de desvirtuar o destruir la existencia de la República Federal Alemana", y el Tribunal Constitucional Federal ha recurrido a esta disposición para prohibir los partidos extremistas, tanto de la izquierda como de la derecha radicales.

Igualmente fundamental en un sistema político moderno es la existencia de elecciones libres, justas y competitivas. Ello no obstante, muy pocas constituciones detallan el procedimiento electoral para elegir al poder legislativo. Las que lo recogen, se limitan en su mayoría a declarar que será "proporcional». La Constitución irlandesa de 1937 representa una notable excepción, y establece en el artículo 16 (2) (5) que el Dai* será elegido según el sistema de representación proporcional por medio del voto único transferible. Este artículo, como el resto de la Constitución, puede reformarse solo mediante referéndum. Durante la posguerra en Irlanda, los gobiernos del Fianna Fail pretendieron en dos ocasiones, en 1959 y 1968, cambiar el sistema electoral, pero las dos veces su propuesta fue rechazada por referéndum. En contraste con Irlanda, la Constitución Francesa de la 5ª República de 1958 no dice nada sobre

13 S. LOW, The Governance of India (T. Fisher Unwin, 1904), pág. 5.

* N. del T.: el Dail es la cámara baja del Oireachtas (el parlamento) de la República de Irlanda. 
el sistema electoral, lo que ha permitido al gobierno modificarlo mediante la legislación ordinaria. Antes de las elecciones parlamentarias de 1986, el Presidente Mitterrand sustituyó el sistema mayoritario de dos vueltas por un sistema de representación proporcional, con el propósito de debilitar a los partidos de la derecha. Sin embargo, la derecha triunfó en las elecciones y, bajo el mandato del Primer Ministro Chirac, restableció el sistema mayoritario de dos vueltas a tiempo para las elecciones legislativas de 1988.

Desde luego estas diferencias entre las constituciones de distintas democracias revelan diferentes experiencias históricas, pero demuestran además que más allá de un núcleo mínimo básico, frecuentemente no hay una frontera clara entre lo que es y lo que no es constitucional. Si una constitución británica tuviera que regular la función de los partidos políticos — ¿idebería incluir las características principales de la Ley sobre Partidos Políticos, Elecciones y Referendos de 2000?; ¿debería exigir que los partidos fueran democráticos en su organización, que fueran transparentes y responsables en su financiación? ¿¿Debería regularse en la constitución el sistema electoral $-\mathrm{y}$, en caso afirmativo, sería solamente el de las cámaras de Westminster, o también los distintos sistemas electorales utilizados para la elección de los órganos descentralizados y en las elecciones locales-? Sin embargo, la cuestión de cuál es el sistema electoral apropiado, tanto para Westminster como para los cargos locales, es ahora, sobre todo, un asunto de discusión política. Es posible que estos sistemas cambien en los próximos años - las autoridades locales en Escocia cambiaron recientemente del sistema uninominal mayoritario a una sola vuelta al método del voto único transferible de representación proporcional, y existe cierta presión en Gales para que las autoridades locales galesas sean elegidas según ese procedimiento. Por otra parte, es previsible que el sistema de elección de los Comunes cambie en un futuro probable-.

La cuestión del sistema electoral plantea además otro problema. Tony Blair prometió que no se modificaría el sistema de elección de la Cámara de los Comunes sin un referéndum; y en un trabajo reciente de investigación, la Biblioteca de la Cámara de los Comunes alega que «según una convención constitucional, los cambios del sistema electoral deben consensuarse hasta donde sea posible entre todos los partidos." Y luego prosigue, en una frase algo confusa, que "esta convención no siempre se ha observado». ${ }^{14}$ ¿Debería esta convención incluirse en la constitución? ¿Debería la constitución disponer que cualquier cambio del sistema electoral de los Comunes requerirá la celebración de un referéndum? Evidentemente, una ley que modificara el sistema electoral que no se sometiera a la aprobación popular no sería invalidada por los tribunales. Pero algunos podrían argumentar que existe hoy un acuerdo generalizado entre la clase política según el cual un cambio en el sistema electoral de la Cámara de los Comunes exigiría un referéndum, a fin de evitar que los gobiernos manipulen el sistema electoral para obtener ventajas polí-

14 House of Commons Library: Standard Note; Speaker's Conference, SN/PC/4426, 12 de septiembre de 2007, pág. 1. 
ticas, como sucedió en Francia. Los redactores de la constitución británica tendrían, por lo tanto, que considerar, en primer lugar, si introducir o no en la constitución el sistema electoral; y, en segundo lugar, si debería haber alguna disposición en el sentido de someter los cambios del sistema electoral al acuerdo de todos los partidos y/o a referéndum.

\section{EL ES Y EL DEBE SER: EL PAPEL DE LAS CONVENCIONES}

El ejemplo del sistema electoral muestra que la promulgación de una constitución británica suscitaría numerosos problemas de considerable dificultad y, además, interrelacionados. En primer término, se presentaría la dificultad de distinguir entre la constitución tal cual es y la constitución cómo pensamos que debería ser; luego, la cuestión de identificar lo que la constitución es de facto, puesto que buena parte de la misma la forman convenciones, cuyos contenido y alcance son a veces poco claros. Finalmente, la cuestión de quién debe poseer la autoridad para identificar lo que es la constitución — ¿una Comisión Real o una especialmente acordada y, en este último supuesto, nombrada o elegida-, o podría encomendarse la labor a un órgano parlamentario?

El borrador de constitución del Institute for Public Policy Research no pretendía ser una codificación de los compromisos británicos vigentes, sino una constitución reformada que esbozaba lo que sus autores consideraron compromisos políticos deseables. En cambio, la constitución elaborada por los estudiantes de nuestro seminario de Oxford y publicada por el Smith Institute y la Revista Political Quarterly, quería ser un codificación de los mecanismos y la forma de funcionamiento actuales. Tratamos de evitar la discusión política sobre el sistema electoral que resultaba más apropiado para la Cámara de los Comunes, el futuro de la Cámara de los Lores, etc. Los Ministros, cuando debaten sobre si hay razones para elaborar una constitución, proponen, presumiblemente, una consolidación del actual estado de cosas más que un sistema de gobierno totalmente reformado.

Pero la distinción entre lo que la constitución es y lo que debería ser no es tan clara como parece a primera vista. El problema es, básicamente, decidir si la constitución debería plasmar la posición legal de manera estricta, "un mero marco, mezcla de leyes y de la prerrogativa real, que presidiera con una dignidad hipotética sobre el mundo real de carne y sangre", una solución que de Smith calificó de "esquizofrenia voluntaria" ${ }^{15}$; o si, por el contrario, debería explicar con detalle la práctica constitucional del momento, lo que significaría tener en cuenta las convenciones constitucionales. Porque la razón fundamental de que no sea fácil a primera vista distinguir entre cuáles son las nor-

15 S. A. DE SMITH, The New Commonwealth and its Constitutions (Stevens, 1964), pág.78. El capítulo tercero de esta obra nos proporciona una explicación excelente de los argumentos en pro y en contra de incorporar las convenciones a la constitución. 
mas constitucionales y cuáles deberían ser, es que esas normas están basadas con frecuencia en convenciones, y no en leyes ni en precedentes judiciales. En un país como los Estados Unidos, con una constitución codificada, cuando se alega que alguna acción es inconstitucional, quiere decirse que la acción es contraria a la constitución. En Gran Bretaña, en cambio, cuando se alega que una acción es inconstitucional, lo que usualmente quiere decirse no es que la acción sea contraria a la ley en sentido estricto, sino contraria a las convenciones. Toda constitución puede basarse hasta cierto punto en convenciones y en leyes. Pero las convenciones jugarán probablemente un papel mayor y más importante en una constitución sin codificar que sea el producto de una larga evolución. Posiblemente habrá menos convenciones en un país como Israel que, como Gran Bretaña, carece de una constitución codificada, pero que en la actualidad está tratando de unificar sus leyes fundamentales en una sola. Israel no fue un Estado hasta 1948 y su historia no es lo bastante larga como para haber adquirido una cantidad considerable de convenciones. En el caso de un país que pretenda elaborar una constitución de novo como ocurrió, por ejemplo, con la Ley Fundamental Alemana de 1949, el problema evidentemente no existe.

La existencia de convenciones plantea dos problemas fundamentales: primero, el de su identificación y distinción de las meras prácticas o de los usos; y, en segundo lugar, si se trata de incluir algunas convenciones en la constitución, el de cuáles deberían incluirse.

Una voz autorizada ha apuntado que, por su naturaleza, es imposible identificar a las convenciones con cierto grado de precisión. «Es...muy difícil trazar una línea entre una práctica obligatoria y otra que no lo es. La característica de las convenciones, es decir, su condición de complemento de las leyes que los tribunales hacen cumplir, parecería imposibilitar una definición precisa de las mismas. ${ }^{16}$ Si esto fuera cierto, sería difícil que la constitución recogiera una declaración precisa de, por ejemplo, los poderes de la Reina. De hecho, sin embargo, las convenciones han cristalizado en otras áreas jurídicas en forma de códigos de prácticas para funcionarios, ministros y miembros del parlamento. Parece inverosímil señalar, por lo tanto, que las convenciones no puedan ser identificadas y declaradas. A decir verdad, los tribunales han reconocido frecuentemente la existencia de las convenciones. En Canadá, el Tribunal Supremo en Reference re Amendment of the Constitution of Canada (1981) fue más allá de ese reconocimiento. Procedió, por vez primera en el contexto del ámbito del common law, a solventar una disputa relativa a si una práctica del pasado - la de asegurar el consentimiento provincial a las enmiendas constitucionales que afectaran al poder de las provincias-, era tanto como una convención, o era meramente un uso. ${ }^{17}$

16 E. C. S. WADE,"Introduction", a la $10^{a}$ edición de la obra Dicey's Law of the Constitution, pág. clv.

17 (1982) 125 D.L.R. (3d.) 1. HOGG, Constitutional Law of Canada, págs.1-26. El Tribunal Supremo resolvió por el voto de la mayoría que la práctica en cuestión equivalía a una convención, pero no era vinculante legalmente. 
En Australia, donde la incorporación de las convenciones sobre el papel de la Reina y del Gobernador General fue primero sugerida por Evatt, se creó una comisión, denominada confusamente "Convención", compuesta por delegados de la Commonwealth y de los gobiernos de los Estados, en los estaban representados todos los partidos, que trató de identificar las convenciones de la constitución australiana. En 1985, su informe estableció una lista de 34 convenciones que regían materias tales como las relaciones entre el Primer Ministro, el Gobernador General y la Cámara de Representantes. El informe iba precedido por la siguiente frase: “Esta Asamblea reconoce y declara que los principios y prácticas siguientes serán observados en Australia como convenciones" ${ }^{18}$.

Un ejercicio de esa naturaleza requiere que, en primer lugar, distingamos entre reglas convencionales y meras generalizaciones relativas al comportamiento político. Por ejemplo, es el caso de que, aunque los proyectos de ley pueden ser presentados en el parlamento por cualquier miembro de una de las cámaras, en la práctica éstos tienen pocas posibilidades de convertirse en leyes, a menos que sean presentados o apoyados por algún ministro. Esto no es, sin embargo, una convención, ya que no es una declaración normativa, sino una generalización sobre el funcionamiento del parlamento, y sería claramente inapropiado incluirla en una constitución. Tampoco seria satisfactorio definir, siguiendo a Wheare, la convención simplemente como "una norma de comportamiento aceptada como obligatoria por aquellos a quienes concierne la vigencia de la constitución". ${ }^{19}$ Los actores políticos pueden equivocarse fácilmente sobre lo que es obligatorio. En 1955, por ejemplo, cuando Sir Anthony Eden, como Primer Ministro, quiso nombrar a Lord Salisbury Ministro de Asuntos Exteriores, fue disuadido por una convención, según la cual el Ministro de Asuntos Exteriores debe pertenecer a la Cámara de los Comunes. ${ }^{20}$ En 1960, sin embargo, Harold MacMillan consiguió nombrar como Ministro de Asuntos Exteriores a Lord Home, de la Cámara de los Lores, y Margaret Thatcher siguió ese precedente en 1979, cuando nombró a Lord Carrington. Por lo tanto, lo que Sir Anthony Eden y otras personas consideraban una convención en determinados círculos políticos, resultó que no era una convención. Si las convenciones son, como ha sugerido una autoridad académica, parte de la "moralidad crítica" ${ }^{21}$ de la constitución, el descubrimiento de lo que son no puede ser una investigación de tipo histórico o sociológico, sino que suscita inevitablemente problemas normativos.

18 Es posible encontrar pormenores de las convenciones en los apéndices al artículo de $\mathrm{CH}$. SAMPFORD y D. WORD, "Codification of Constitutional Conventions in Australia [1987] P.L. 239240. Véase también C. SAMPFORD, "Recognise and Declare'. An Australian Experiment in Certifying Constitutional Convencions" (1987) 7 O.J.L.S. 369-417; y H. V. EVATT, The King and His Dominion Governors (Oxford University Press, 1936).

19 WHEARE, Modern Constitutions, pág.122. (N. del T.: op. cit. en español, pág. 128).

20 The Earl of Avon, (Sir Anthony Eden), Full Circle (Cassell, 1960), pág. 274.

21 G. MARSHALL, Constitutional Conventions (Oxford University Press, 1984) pág. 210. Esta obra contiene un análisis incisivo de los problemas que surgen de la existencia de las convenciones constitucionales en el Reino Unido de la Gran Bretaña. 
Necesitamos, por lo tanto, distinguir, como hizo el Tribunal Supremo en Canadá, entre convenciones y meros usos. Pero, una vez que hayamos identificado las convenciones, debemos considerar hasta que punto habría que incluirlas en la constitución. Seguramente, sería erróneo limitarse a decir, en relación con la función legislativa del jefe del Estado, que la "Reina-en-el-Parlamento"* sanciona la ley, pues ello implicaría que la Reina es una parte real en el procedimiento legislativo. Parecería más sensato sostener que el Soberano normalmente da su consentimiento a la legislación presentada por el gobierno, aunque pueda surgir algún desacuerdo cuando se trate de especificar las precisas circunstancias bajo las cuales el soberano no necesita sancionar la legislación. Por ejemplo, chabría Jorge V actuado constitucionalmente de haberse negado a sancionar el proyecto de Ley del Gobierno de Irlanda de 1914, que preveía un parlamento para Irlanda fruto del autogobierno? Ciertamente así lo pensó, y también autoridades en materia de Derecho constitucional de la talla de Dicey y Anson. A decir verdad, Anson, autor de la reconocida obra The Law and the Custom of the Constitution, escribió al The Times, en septiembre de 1913, justificando la utilización del veto real. ${ }^{22}$ Podría, no obstante, acordarse que se insertara en la constitución un precepto en el que se dispusiera que el Soberano normalmente sanciona la legislación. Más difícil de solventar serían algunas otras materias relativas a las competencias del Jefe del Estado. Por ejemplo, bajo qué circunstancias está legitimada la Reina para rechazar una petición de disolución —un problema que adquiriría una significación singular en el caso de un parlamento en el que ningún partido contase con la mayoría de los escaños; más aún, en el supuesto de que se adoptara el sistema proporcional para las elecciones a la Cámara de los Comunes, en cuyo caso cada vez que se constituyera el parlamento, el resultado sería un parlamento sin un partido político mayoritario-.

¿Qué sucede con las convenciones en otras áreas de gobierno? Según dice Jennings, sería "una norma constitucional singular, que menciona al Gabinete, porque a él se refiere la Ley de los Ministros de la Corona, de 1937, pero no puede decir lo que hace... Es una norma 'constitucional' que dice muy poco de la constitución. ${ }^{23}$ ¿Debería la constitución recoger la convención Sewel, de que el Parlamento "normalmente no legisla en materias "devueltas" a Escocia sin el consentimiento del Parlamento de Escocian? ¿24 Deberían precisarse las competencias de la Cámara de los Lores? ¿Cuáles son? En la ley, la

* N. del T.: la expresión Queen in Parliament (Reina en el Parlamento) es una expresión constitucional británica con la que se hace referencia a la Corona en el ejercicio de sus competencias legislativas, para lo cual cuenta con el asesoramiento y el consentimiento de las dos cámaras parlamentarias.

22 Véase V. BOGDANOR, The Monarchy and the Constitution (Oxford University Press, 1995), pág.122-135, La carta de Anson aparece reproducida en I. JENNINGS, Cabinet Government,

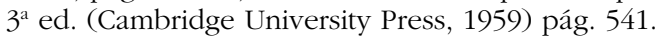

23 I. JENNINGS, The Law and the Constitution, $5^{\mathrm{a}}$ ed. (University of London Press, 1959), págs. 70-71.

24 Hansard, HL Vol.592, col. 791 (21 de julio de 1998). 
respuesta está clara. Los Lores solo tiene competencia sobre los proyectos de ley no financieros, que pueden demorar durante una sesión, y sobre un proyecto de ley para prorrogar el mandato del parlamento, para lo cual conservan un veto absoluto. Tienen también poder absoluto para rechazar la legislación secundaria, pues las Leyes del Parlamento regulan sólo la legislación primaria - la legislación secundaria era mínima en el tiempo en que se aprobó la Ley del Parlamento de 1911—. * Pocos, sin embargo, aceptarían como una afirmación sensata sobre el sistema constitucional que los Lores tengan poder absoluto para rechazar la legislación secundaria. Así pues, las atribuciones de la Cámara de los Lores no solo están limitadas por ley, sino también por convención.

La convención Salisbury, formulada en 1945 por un acuerdo entre el partido conservador y el laborista - fue la primera vez desde la Ley del Parlamento de 1911 en que la Cámara de los Lores, entonces dominada por los conservadores, tenía ante sí un gobierno mayoritario de izquierda-, establecía que los Lores no se oponen a proyectos de ley presentados por el gobierno, que formasen parte de las medidas incluidas en su programa político, en la segunda o tercera lectura que se realice de dichos proyectos en la cámara*.25 También por convención, los Lores normalmente no rechazan la legislación secundaria. De ese modo, parece que, así como en la sección relativa al Jefe del Estado debería incluirse la convención que establece que la Reina normalmente no rechaza (no se niega a sancionar) las leyes que le presenta el gobierno, también en la sección relativa a la Cámara de los Lores deberían incluirse convenciones, además de normas legales.

No obstante, podría resultar complicado incluir la convención Salisbury en un texto constitucional, y ello por dos razones. La primera, debido a que, por su naturaleza, resulta altamente flexible en lo que a su contenido se re-

* N. del T.: Las dos principales categorías de normas en el ordenamiento jurídico británico son la "primary legislation" (legislación primaria) y la "secondary legislation" (legislación secundaria). La primary legislation es el término general que se utiliza para hacer referencia a la mayoría de los tipos de leyes que aprueban los órganos legislativos (parlamentos), también llamados statutes, así como a ciertas normas aprobadas por la Corona. La secondary legislation, también llamada subordinate o delegated legislation, agrupa todas aquellas normas aprobadas por un órgano diferente del Parlamento, normalmente un órgano ejecutivo, de acuerdo con una competencia concedida por una norma de la legislación primaria, y cuyo objeto es desarrollar o ejecutar el contenido de dicha norma de la legislación primaria.

Debido a su naturaleza, las normas de la legislación secundaria están sometidas a controles parlamentarios; por regla general, o bien se exige el voto afirmativo de las dos cámaras antes de la aprobación de la norma, o bien se somete dicha norma, una vez aprobada, a las cámaras, que pueden presentar un veto contra la misma.

* N. del T.: En la mayoría de los procedimientos legislativos del Parlamento Británico se realizan, en cada una de las dos cámaras, tres lecturas de los proyectos de ley que se presentan. La primera lectura se celebra al entrar el proyecto en la cámara; la segunda lectura, con debate y votación, antes de remitir el proyecto a la correspondiente comisión; y la tercera, para su debate y votación final, a partir del trabajo realizado por la comisión.

25 Royal Commission on Reform of the House of Lords, A House for the Future, 2000, Cm. 4534, para. 4.21 . 
fiere y, por consiguiente, es difícil de exigir su estricto cumplimiento. De hecho, ésta fue la conclusión a la que llegó en 2005 una Comisión Mixta sobre Convenciones creada para analizar la posibilidad y la factibilidad de codificar las diversas convenciones relativas a las relaciones entre las dos cámaras parlamentarias, incluida la convención Salisbury. Su informe final, Conventions of the UK Parliament, ${ }^{26}$ contiene material valioso sobre las dificultades de definir con precisión las convenciones que regulan la actividad de los Lores. La Comisión declaró ${ }^{27}$ que la convención Salisbury había evolucionado desde 1945 y que ahora debería denominarse "convención sobre los proyectos de ley gubernamentales" (Government Bill Convention). También afirmó ${ }^{28}$ que los Lores no deberían rechazar legislación secundaria, salvo en circunstancias excepcionales. Sin embargo, no quiso definir cuáles podrían ser esas circunstancias. La Comisión concluyó que:

"Todas las recomendaciones que se hagan en el sentido de formular o codificar las convenciones están condicionadas por la concepción, de todos conocida, de que las convenciones, como tales, son flexibles, y su cumplimiento es difícil de exigir, especialmente en un ámbito de autorregulación como el de la Cámara de los Lores". ${ }^{29}$

La segunda dificultad, que la Comisión no advirtió en toda su extensión, deriva de que la convención Salisbury se ha convertido hoy en día en objeto de discusión entre los partidos políticos. Esta convención ha sido aceptada sin apenas haber sido cuestionada hasta la aprobación de la Ley de la Cámara de los Lores de 1999; pero actualmente hay quienes argumentan que esta Ley elimina la base sobre la que se apoya la citada convención, ya que la Ley establece que todos los pares hereditarios, salvo 92 de ellos, dejarán de pertenecer a la Cámara de los Lores. En consecuencia, ya no habrá una mayoría permanente del partido conservador entre los Lores. En el momento en el que esto se escribe, es el partido laborista el que tiene el grupo más grande entre los Lores, aunque sin una mayoría absoluta. De hecho, parece haberse desarrollado una nueva convención de acuerdo con la cual, el grupo de un único partido político no debe nunca más disfrutar de una mayoría absoluta entre los Lores.

Como resultado de este desarrollo, los Liberal-demócratas argumentan ahora que la convención, que tiene su origen en un acuerdo en el que ellos no tomaron parte, ha servido para regular las relaciones parlamentarias sólo cuando la Cámara de los Lores representaba prácticamente a un único partido político. A partir de ahora, que ya no va a darse nunca más ese caso, consideran que debería reemplazarse dicha convención por una nueva, más adecuada a la situación actual. Lord McNally, líder de los Liberal-demócratas en la Cámara de 
los Lores, argumentaba, en un debate celebrado en la misma el 26 de enero de 2005, que "la convención Salisbury fue diseñada para proteger a un gobierno no conservador de un posible bloqueo por parte de una Cámara de los Lores con una mayoría de base hereditaria. No fue diseñada para proporcionar más poder a algo contra lo que el último Lord Hailsham correctamente previno, y que él consideraba una dictadura electiva localizada en otro lugar contra los pesos y contrapesos legítimos ejercidos por esta segunda cámara ${ }^{30}$. En un debate posterior, este líder declaró: "yo no creo que una convención diseñada hace 60 años sobre las relaciones entre una cámara dominada completamente por un grupo conservador hereditario y un gobierno laborista que tenía el respaldo del 48 por ciento de los votos deba aplicarse de la misma manera a la situación en la que actualmente nos encontramos ${ }^{31}$. El gobierno, por el contrario, arguye que la convención Salisbury todavía debe respetarse, dado que la razón de ser de esta convención, desde su punto de vista, reside en que la Cámara de los Lores es un órgano no electivo, y no simplemente en que es una cámara permanentemente dominada por un único partido.

Así pues, no siempre está claro si existe una convención en una materia determinada, ni qué obligaciones derivan de ella. Un ejemplo, que constituyó un excelente ejercicio para los estudiantes de nuestro seminario en Oxford, es el relativo a si la votación que tuvo lugar en la Cámara de los Comunes del 18 de marzo de 2003 antes de la guerra de Irak ha creado una convención, de tal modo que, en el futuro, el gobierno estaría actuando inconstitucionalmente si quisiera desplegar tropas sin el apoyo parlamentario. Durante el debate previo a la votación, Jack Straw, Ministro de Asuntos Exteriores, declaró que era "Constitucionalmente propio de una democracia moderna" que el gobierno solicitase el apoyo explícito de la Cámara de los Comunes para llevar a cabo una acción militar». Los estudiantes, después de cierta discusión, consideraron que este único precedente había creado una convención y que ésta debía incluirse en la constitución. ${ }^{22}$

Cuando no resulta claro si una convención existe, o cuál es su ámbito de aplicación, quizás el camino más seguro sea, a diferencia de lo dicho sobre la sección relativa al Jefe del Estado, incorporar al texto constitucional solamente las normas legales, dejando la determinación precisa de las convenciones a los actores políticos. Pero esto no sería totalmente satisfactorio. Así, en el caso de la Cámara de los Lores, si solo se incorporase la norma legal, podrían parecer legítimos los argumentos de aquellos que, como Lord McNally, consideran que dicha Cámara ya no debería sentirse vinculada por la convención Salisbury. Esto podría permitir a los Lores, actuando en el ámbito de sus facultades, convertir en inútil el último año del mandato de un gobierno, desde el punto de vista legislativo. Más aún, una constitución que no contuviera la convención de acuerdo con la cual los Lores normalmente no rechazan la le-

30 Hansard, HL Vol. 668, col. 371 (26 de enero de 2005).

31 Hansard, HL Vol. 672, col. 20 (17 de mayo de 2005).

32 Hansard, HC Vol. 401, col. 900 (18 de marzo de 2003). 
gislación secundaria podría estar legitimando el uso de este arma jurídica, y ello supondría una intromisión aún mayor y más peligrosa en las facultades del gobierno. Ello no obstante, si los Lores se mantuviesen firmes en su voluntad de rechazar la normativa del gobierno, seguramente se les podría acusar de actuar inconstitucionalmente. Pero resultaría difícil defender esta acusación con un texto constitucional que solamente contuviese las normas legales y no incluyese la convención según la cual los Lores no rechazan la legislación secundaria. Si se excluyesen de dicho texto las convenciones que se refieren a la Cámara de los Lores, la constitución resultante contribuiría más bien poco a clarificar las funciones y competencias concretas de la cámara alta.

Las convenciones, por lo tanto, pueden ser muy generales y su interpretación no resulta siempre ni mucho menos obvia. Se podría objetar que lo mismo ocurre en muchas ocasiones con las normas escritas; pero éstas, sin embargo, pueden ser interpretadas por los tribunales. A diferencia de la experiencia canadiense, en el caso de las convenciones en el Reino Unido no existe un árbitro similar al que existe en aquel país. Si la interpretación de una norma convencional no es clara, su determinación final suele depender de la evolución del contexto político más que del pronunciamiento de un tribunal. Por ejemplo, el ejercicio de las prerrogativas personales del Jefe del Estado se ha visto seriamente limitado por el desarrollo de un sistema bipartidista, ya que éste ha supuesto que la Reina no ha tenido que utilizar su ámbito de discrecionalidad a la hora de elegir al Primer Ministro ni tampoco a la hora de aceptar una propuesta de disolución parlamentaria elevada por el gobierno. Habiendo sólo dos partidos, generalmente resulta evidente cuál debe ser la respuesta de la Reina. Aunque esto podría cambiar en el caso de que existiese un parlamento multipartidista en el que ya no fuesen predecibles las decisiones; así, en el caso de que la Cámara de los Comunes pase a elegirse mediante un sistema proporcional, el parlamento resultante probablemente sería de ese tipo, ya que desde 1935 ningún gobierno ha conseguido el 50 por ciento de los votos. Esto modificaría significativamente el papel de la Reina. ${ }^{33}$

Puede parecer que un texto constitucional promulgado que codifique convenciones trae consigo una juridificación considerable de los acuerdos de carácter constitucional. Lo que en el pasado ha sido esencialmente político, podría ahora convertirse en una cuestión de derecho constitucional. Así, en teoría podría ocurrir que tuviesen que ser los tribunales quienes decidieran la interpretación de las convenciones. Éstas ya no dependerían más de las vicisitudes políticas, sino que se convertirían en "justiciables". Pero esto plantea problemas de enorme calado. ¿Pueden realmente los tribunales decidir, por ejemplo, qué deriva del principio de la responsabilidad ministerial? ¿ello supondría que los tribunales podrían concretar en qué casos existe la obligación de revelar información ministerial al parlamento, y en qué circunstancias los

33 Vid. V. BOGDANOR, Multi-Party Politics and the Constitution, (Cambridge University Press, 1983). 
ministros tienen la obligación de dimitir después de cometer un determinado fallo? Si es así, los tribunales pasarían a ocupar un lugar en los procesos políticos mucho más central del que nunca han ocupado hasta ahora. Sin embargo, los tribunales que tuviesen que enfrentarse a cuestiones de este tipo seguramente se resistirían a participar en este tipo de procesos políticos y probablemente se acogerían a la doctrina de los "actos políticos", que el Tribunal Supremo de los Estados Unidos ha utilizado frecuentemente, aunque no siempre, cuando ha tenido que enfrentarse a cuestiones relativas a la teoría de la separación de poderes. Los tribunales alemanes también han desarrollado una doctrina similar que permite a los órganos judiciales inhibirse en ciertos casos.

Las convenciones, sin embargo, pueden ser de muy diferente tipo. Algunas pueden ser fundamentales, como, por ejemplo, la convención de acuerdo con la cual el gobierno debe presentar su dimisión después de haber perdido una moción de confianza en la Cámara de los Comunes; la interpretación de otras convenciones, como, por ejemplo, la relativa a la responsabilidad individual de los ministros, aunque quizás sea igual de fundamental que la anterior, puede evolucionar con el tiempo y su interpretación puede resultar algo menos clara; otras convenciones, como, por ejemplo, aquella conforme a la cual los miembros de la Familia Real no pronuncian discursos que tengan implicaciones para los partidos políticos, son menos fundamentales y simplemente señalan la inconveniencia de una determinada actuación. ${ }^{34}$

En un informe presentado a la Convención Constitucional Australiana, Cheryl Saunders y Ewart Smith también realizaron una distinción entre convenciones de diferente tipo:

"Algunas convenciones resultan muy apropiadas para ser incluidas en una constitución escrita y para que su cumplimiento pueda exigirse ante los tribunales; otras, pueden ser incluidas en la constitución, pero como declaraciones de principios, sin que puedan utilizarse ante los tribunales; otras, pueden articularse fuera del texto constitucional mediante un acuerdo informal sobre lo que debe entenderse que es su contenido". 35

Desafortunadamente, en ese informe no se indica cuáles son las convenciones que pertenecen a cada una de estas tres categorías.

Lo que parece claro es que sólo algunas convenciones son adecuadas para ser incorporadas a un texto constitucional y para ser "justiciables", es decir, para que se pueda exigir su cumplimiento ante los tribunales. También podemos admitir la posibilidad de incluir una convención pero excluyendo

34 A. HEARD, en Canadian Constitutional Conventions: The Marriage of Law and Politics (Toronto: Oxford University Press, 1991), pág. 141, realiza una triple distinción de este tipo entre diferentes convenciones.

35 "Identifying Conventions Associated with the Commonwealth Constitution", Australian Constitutional Convention, Standing Committee "D", vol.2, 1982, pág.1, citado en HEARD, Canadian Constitutional Conventions: The Marriage of Law and Politics, pág. 151. 
expresamente que se pueda exigir su cumplimiento mediante una acción ante los tribunales; tal y como ocurre con las actuaciones del Speaker (el presidente de la cámara) relativas a un proyecto de ley financiero, a los efectos de lo establecido en la Ley del Parlamento, que no pueden ser cuestionadas ante un tribunal. Aunque no sea sencillo separar lo que es político de lo que es constitucional o jurídico, ciertamente sí es posible hacerlo, tal y como ha demostrado la experiencia de otras jurisdicciones.

En este punto, sin embargo, un crítico hostil podría plantear la siguiente cuestión: si al menos algunas de las convenciones no van a ser "justiciables", es decir, no se va a poder exigir su cumplimiento ante los tribunales, ¿qué sentido tiene entonces incorporarlas a un texto constitucional? La respuesta solo puede ser la de que esa incorporación contribuiría a dar mayor claridad a un área jurídica que con frecuencia resulta confusa, y, por ello, serviría como ayuda en la resolución de crisis constitucionales. No obstante, la promulgación de un texto constitucional puede conducir perfectamente a una división entre tres tipos diferentes de normas convencionales: normas incorporadas al texto y "justiciables", normas incorporadas al texto pero no "justiciables", y normas que no se han incorporado al texto pero que son en última instancia vinculantes. En el caso de que una norma convencional haya sido incorporada el texto de la constitución pero se haya declarado su carácter de "no justiciable", podría plantearse la cuestión de qué autoridad tendría un tribunal para pronunciarse sobre ella y qué fuerza tendría su pronunciamiento dado que no podría atribuir consecuencias jurídicas a la convención aplicable al caso.

Además de lo anterior, el argumento de acuerdo con el cual la constitucionalización de una convención contribuiría a dotar de mayor claridad a un ámbito determinado debe tomarse con ciertas precauciones, puesto que la convención tendría que recogerse en términos muy generales y su interpretación seguiría siendo objeto de controversia. La promulgación de un texto constitucional no resolvería la dificultad fundamental, que estriba en que la decisión relativa a la existencia o no de una convención, tal y como hemos visto en el caso de la convención Salisbury, no es en modo alguno una actividad puramente intelectual o jurídica, sino una actividad esencialmente política. Donde quiera que se discuta sobre si una práctica concreta constituye una convención y sobre qué es lo que prescribe exactamente, no se trata solo de un debate intelectual del mismo modo en que no lo es el debate sobre el "significado real" de la cláusula de comercio o del principio de igualdad en la Constitución de los Estados Unidos; tampoco se trata de un debate sobre una cuestión de hecho, sobre si los actores políticos se sienten de hecho obligados; se trata de un debate sobre si ellos deben sentirse obligados. Es una discusión sobre lo que términos tales como "igualdad" deben significar, así como sobre lo que de hecho significan. De esta forma, la discusión sobre la vigencia de la convención Salisbury es algo más que simplemente un debate intelectual, ya que es, en parte, una discusión sobre la localización exacta del poder político. Por ese motivo es posible que no exista una forma totalmente 
satisfactoria de determinar quién tiene la razón en este tipo de discusiones, ya que la respuesta puede depender del equilibrio de los poderes políticos, de las vicisitudes políticas y del estado de la opinión pública. ${ }^{36}$ Esta es la razón por la que John Griffith, como es bien conocido, insiste en que la constitución británica es "ni más ni menos que lo que ocurre». ${ }^{37}$ De acuerdo con nuestro peculiar sistema no codificado, frecuentemente sucede que, allí donde son aplicables las convenciones, los límites de la constitución tienden a coincidir con los límites del poder político. En un período de cierta agitación constitucional, como está ocurriendo ahora en el Reino Unido, es particularmente difícil predecir los resultados de lo que en esencia es una contienda política, y, por lo tanto, es difícil encontrar una solución satisfactoria al problema de incorporar a un texto constitucional, por ejemplo, las funciones y competencias de la Cámara de los Lores.

\section{ELABORACIÓN, RATIFICACIÓN Y MODIFICACIÓN}

El problema de determinar cómo vamos a decidir qué convenciones deberían incluirse en un texto constitucional y cómo deberían formularse conduce a una cuestión aún de mayor calado. ¿A quién se ha de reconocer la autoridad para tomar las decisiones en esta materia? Cabe entender que un documento constitucional no pueda ser elaborado del mismo modo que se aprueba una ley ordinaria. Sin embargo, el informe de la Convención Constitucional Australiana, ${ }^{38}$ valioso como es, plantea la cuestión de cuál es la autoridad de la que puede disfrutar una constitución establecida por una comisión o asamblea, a pesar del reconocido prestigio que ella o sus miembros pudieran tener. Así, con objeto de que ésta tuviera suficiente legitimidad, se exigiría presumiblemente que en la asamblea constituyente existiese representación de todos los partidos políticos, como ocurrió en el caso de Australia. Supongamos, sin embargo, que no se lograse un acuerdo por unanimidad sobre si existe una determinada convención, como sucede, por ejemplo, respecto a la convención Salisbury. Esa falta de unanimidad ímpediría que se le siguiera considerando como una convención? ¿Podrían los representantes de un partido político, aunque fueran una minoría, obtener así un poder de veto y ejercerlo, o se admitiría que la decisión fuera tomada por mayoría o por una mayoría cualificada? Algunas convenciones en el informe Australiano fueron, de hecho, decididas por la mayoría. De otro lado, ¿cómo se modificarían las convenciones una vez incorporadas al texto constitucional? Las respuestas a estas preguntas no son ni mucho menos sencillas. Podría parecer que la Convención Australiana tuvo éxito al crear una norma de reconocimiento para

36 Vid., sobre esta cuestión, G. C. MOODIE, "The Monarch and the Selection of a Prime Minister: A Re-Examination of the Crisis of 1931" (1957) 5 Political Studies, págs. 18-19.

37 J. GRIFFITH, "The Political Constitution" (1979) 42 M.L.R. 1, en la pág. 19.

38 Vid. supra nota 35. 
las convenciones, pero no creó una norma para las modificaciones. Existiría de este modo, cierto peligro en crear "prácticamente un grado casi único de inflexibilidad, restringido a la indeterminación y falta de efectividad, y carente de justiciabilidad". ${ }^{39}$

Una asamblea formada por "los mejores" (the great and the good") no es desde luego la única solución organizativa para constituir un órgano en el que se elaboren y discutan las propuestas de constitución. En Gran Bretaña actualmente se está tomando en consideración la propuesta de que se cree una Convención cuyos miembros sean elegidos directamente por los ciudadanos. Los Demócratas-liberales recientemente han planteado la posibilidad de que la mitad de los miembros de esa Convención sean elegidos mediante una especie de sorteo al azar. ${ }^{40}$ Otros, en cambio, consideran que todos sus miembros deberían ser elegidos. El peligro que encierran propuestas de este tipo es que, dado que la mayoría de las personas atribuye una relevancia relativa a los asuntos constitucionales, la participación en las elecciones sería baja y, en consecuencia, la Convención podría presentar un déficit de legitimidad.

Otra alternativa es crear una Convención Constitucional representativa, pero cuyos miembros no tengan que ser elegidos, análoga a la que funcionó en Escocia entre 1989 y 1995..$^{41}$ Esta Convención estaba formada por representantes de los dos partidos mayoritarios, el laborista y el demócrata-liberal, junto con representantes de otros partidos minoritarios, así como de las principales instituciones de la sociedad civil escocesa - por ejemplo, las Iglesias y los sindicatos-. La Convención escocesa, sin embargo, se estableció para decidir sólo sobre una cuestión, además bastante concreta, como fue la "devolución" de competencias de Inglaterra a Escocia; a lo que hay que añadir que estaba compuesta sólo por partidarios de dicha devolución, ya que los conservadores y el SNP renunciaron a formar parte de ella. De otro lado, Escocia dispone de una sociedad civil mucho más cohesionada que la de Inglaterra, y sería mucho más sencillo lograr un cierto consenso allí, especialmente si se trata de cuestiones relacionadas con Escocia y sus nacionales. Una Convención Constitucional que cubriera toda Gran Bretaña tendría que ocuparse de una gran variedad de cuestiones muy diferentes y sería mucho más difícil que en Escocia garantizar una representación equilibrada de las distintas corrientes de opinión sobre cada una de las materias. Si todos los partidos políticos estuviesen de acuerdo en participar en la Convención, en ella se podría reproducir la lucha de poder entre partidos que tiene lugar en Westminster y contribuiría a producir una mayor alienación. Si, por el contrario, algunos de los

39 SAMPFORD, (1987) 7 O.J.L.S. 369, en la pág. 403. La referencia a la norma de reconocimiento se ha tomado, obviamente, de H. L. A. HART, The Concept of Law (Clarendon Press, 1961).

40 Vid. M. CAMPBELL, "A Rescue Plan for Politics", Guardian, 6 de septiembre de 2007.

41 Vid. V. BOGDANOR, Devolution in the United Kingdom (Oxford University Press, 1999), págs. 196 a 198, y K. WRIGHT, The People Say Yes: The Making of Scotland's Parliament (Argyll Publishing, 1997). K. WRIGHT fue Presidente de la Convención. 
partidos en la oposición renunciasen a estar representados en ella, como ocurrió en Escocia, la Convención podría ser considerada simplemente como un instrumento del partido en el gobierno.

Es posible pensar en una Convención compuesta por ciudadanos no expertos, aunque hayan sido elegidos para ello, no sería el órgano más adecuado para presentar propuestas dirigidas a elaborar y promulgar un texto constitucional, ya que realmente han sido pocas las propuestas concretas sobre este asunto que se han formulado desde la ciudadanía. Quizás fueran necesarios cierto apoyo y guía en la materia, y, para ello, la participación en el proceso de "los mejores" parece inevitable. Una manera de conseguir esta participación, al mismo tiempo que se mantiene la contribución de los ciudadanos, sería iniciando una consulta y un ejercicio preparatorio a través de una Comisión Real. Los miembros de esa Comisión tendrían que ser expertos nombrados por el gobierno, pero indudablemente éste debería buscar el apoyo de los principales partidos en la oposición para las personas que fueran a ser nombradas.

La gran ventaja de una Comisión de este tipo es precisamente que puede servir como ejercicio preparatorio. Debería recabar datos y declaraciones, escritas y orales, en diferentes partes del Reino Unido; indudablemente, sus sesiones orales deberían ser televisadas, y tendría que ser objeto de una publicidad considerable tanto en los medios locales como nacionales. Ello contribuiría a dar mayor relevancia a las cuestiones relacionadas con la democracia y haría que los ciudadanos se planteasen dichas cuestiones. Entre 1969 y 1973 la Comisión Real Kilbrandon sobre la Constitución, ${ }^{42}$ que se ocupó principalmente de la "devolución", realizó sesiones informativas en diferentes partes del país y esto sirvió para que aumentase considerablemente el interés por el tema de la "devolución".

Una vez que se ha elaborado una constitución, se necesita diseñar un procedimiento para su ratificación. El preámbulo de la Constitución de los Estados Unidos comienza del siguiente modo: "We the people" (nosotros, el pueblo), con lo que quiere decir que el pueblo de los Estados Unidos se ha dado a sí mismo esa constitución. Sin embargo, el pueblo no fue consultado ni respecto a la elección de los Padres Fundadores, ni a la hora de la ratificación de la constitución que elaboraron los representantes elegidos para ello. De forma similar, el preámbulo de la Constitución alemana de 1949 declara que «el pueblo alemán ha adoptado, en virtud de su poder constituyente, esta Ley Fundamental", aunque esa constitución también fue ratificada por representantes elegidos para ello y no directamente por los ciudadanos. En algunos países, no obstante, como en Francia, por ejemplo, la constitución ha sido ratificada directamente por los ciudadanos.

En la actualidad el referéndum es un procedimiento aceptado en Gran Bretaña, a pesar de que solo se haya celebrado un único referéndum de ámbito nacional —el que se celebró en 1975 para decidir si Gran Bretaña per- 
manecería en las Comunidades Europeas- Sin embargo, en numerosas ocasiones se ha prometido la celebración de consultas populares de este tipo, y existe un acuerdo general en que la incorporación a la "Zona Euro" y la modificación del sistema electoral para elegir a los miembros de la Cámara de los Comunes son decisiones que exigirán para su aprobación la celebración de un referéndum. Sobre la necesidad de referéndum en otras materias existe mayor discusión. En el momento en el que se escribe este artículo, por ejemplo, se ha generado cierto debate sobre si la ratificación del Tratado Europeo actualmente propuesto exigiría la participación del pueblo mediante referéndum. Así pues, no existe un acuerdo general sobre el papel preciso de los referendos. No obstante, sería lógico proponer que la aprobación de un texto constitucional fuese ratificada por los ciudadanos mediante un referéndum. Si esto ocurriera, el parlamento habría expresado de la forma más clara posible que en el futuro ya no sería nunca más soberano, puesto que quedaría vinculado por una constitución que habría sido promulgada, no sólo por él mismo, sino también por el pueblo, y de la que derivarían tanto sus funciones y competencias, como los límites de las mismas. ${ }^{43}$

¿La reforma de la constitución también debería estar sujeta a referéndum o podría el parlamento modificarla por sí mismo? Y, si el parlamento tuviera ese poder de reforma ¿podría modificar la constitución de la misma manera que aprueba otro tipo de leyes, o debería exigirse un procedimiento especial para ello, como, por ejemplo, una modificación explícita o una mayoría cualificada? En el ejercicio que realizamos con alumnos en el otoño de 2006, se les propuso reflexionar y decidir cuál consideraban que era el método de reforma más apropiado para la constitución, tal y como ésta era entonces, no tal y como les gustaría que fuese. Los estudiantes presentaron tres posibilidades. La primera consistía en que la constitución, del mismo modo que sucede con la mayoría de las normas, pudiera ser modificada de forma implícita y que una norma posterior pudiera "prevalecer" sobre ella y derogarla; que pudiera, por lo tanto, ser tratada exactamente como si fuese una norma ordinaria. La segunda opción consistía en que la constitución solo pudiera ser modificada de forma explícita, tal y como sucede con la Ley de las Comunidades Europeas y con la Ley de Derechos Humanos. La tercera consistía en reconocer a la constitución una rigidez especial de manera que solamente pudiera ser modificada a través de un proceso que exigiese mayoría cualificada en el parlamento y la celebración de un referéndum. La mayoría cualificada exigiría el voto favorable de al menos dos tercios de los miembros de cada cámara, siempre que estuviesen presentes en la votación al menos el 50 por ciento de los miembros de cada una de ellas. Después de un debate sobre las tres opciones, los alumnos tuvieron que decidir, por votación, la opción que consideraban que estaba más en consonancia con la práctica actual. Por una pequeña diferencia, optaron por la segunda alternativa, con el requisito de que

43 Cfr. O. H. PHILLIPS, Reform of the Constitution (Chatto and Windus/Charles Knight, 1970), pág. 156. 
la modificación fuese expresa. Así pues, cualquier reforma de la constitución debería presentarse expresamente a través de un proyecto de modificación constitucional. Ese proyecto, no obstante, se discutiría y aprobaría de acuerdo con el procedimiento legislativo ordinario. Aunque otra opción sería la de atribuir a la Cámara de los Lores un poder de veto absoluto sobre un proyecto de reforma constitucional, similar al que posee respecto a los proyectos que se pueden presentar para prorrogar el mandato de un parlamento, argumentando que este caso proporciona un precedente para otras leyes que tengan una especial relevancia constitucional.

Los alumnos también decidieron que debía haber otras tres restricciones a la soberanía del parlamento. La primera, que "los tribunales no aplicarán ninguna norma legal que sea incompatible con el derecho europeo de efecto directom. La segunda, que se exigirá un referéndum previo a la constitución de cualquier órgano producto de la "devolución", directamente elegido, y con competencias legislativas o ejecutivas. La tercera, que cualquier modificación constitucional que tuviera por objeto la reforma o la supresión de la concreta previsión anterior exigirá un referéndum antes de poder entrar en vigor. Se puede argumentar que las primeras dos restricciones son el reflejo de acuerdos actualmente vigentes, mientras que la tercera es necesaria en orden a proteger la segunda de ellas.

Naturalmente, si se toma realmente en serio el principio de la soberanía del parlamento, no parece que tenga mucho sentido promulgar un texto constitucional. Si el parlamento es soberano, la constitución británica puede resumirse en exactamente las siguientes palabras: lo que la Reina en el Parlamento promulgue es derecho. Sin embargo, el primer objetivo de promulgar una constitución es establecer, a través de una especie de derecho "superior", prescripciones que resulten más difíciles de modificar que las que pueda contener una ley ordinaria. Una constitución, por ello, haría realidad jurídicamente la afirmación de que el parlamento ha abdicado de su soberanía ${ }^{44}$ En The Law of the Constitution, Dicey descarta el:

«...extraño dogma... de que un poder soberano, como el del Parlamento del Reino Unido, no pueda, a través de un acto de él mismo, renunciar a su propia soberanía. ... Argumentar o deducir que, puesto que la soberanía no es limitable (lo cual es cierto), no puede someterse (lo que es evidentemente incierto), supone confundir dos ideas muy diferentes. Es lo mismo que argumentar que, puesto que ningún hombre puede, mientras vive, renunciar a hacer lo que quiera, a su libre albedrío, entonces ningún hombre puede cometer suicidio".

La soberanía, afirma Dicey, puede renunciar por sí misma a su autoridad transfiriendo de forma permanente parte de esa autoridad a otra persona u órgano. Si, por ejemplo, las Leyes de la Unión de 1706, aprobadas por los Par-

44 Debemos esta idea al professor Anthony Bradley, aunque, por supuesto, él no es responsable del uso que hacemos de ella. 
lamentos de Inglaterra y Escocia, hubieran mantenido activos los Parlamentos de Inglaterra y Escocia con el único propósito de modificar, si fuera necesario, esas Leyes, y hubieran otorgado al nuevo Parlamento de Gran Bretaña, creado por esas mismas Leyes, el poder para aprobar cualquier tipo de ley, excepto una ley que modificase, incumpliese o suprimiese las Leyes de la Unión, entonces esas Leyes habrían sido leyes fundamentales inmodificables jurídicamente por el nuevo Parlamento de Gran Bretaña. ${ }^{45}$

Quizás el interés por elaborar y promulgar un texto constitucional sea consecuencia, precisamente, de que hoy en día algunos consideran que el parlamento ya no es soberano, o, desde otra perspectiva, consecuencia de que la soberanía parlamentaria ya no parece actuar "sobre todas las personas, materias y cosas", lo que antes parecía posible, tal y como mostraba la sección 75 de la Ley del Gobierno de Irlanda de 1920, una norma declarativa que establecía (equivocadamente, como uno podría sospechar) que Westmister continuaría teniendo competencias sobre Irlanda del Norte, después de que se estableciera un parlamento legislativo en ese territorio. ${ }^{46}$ Pero la principal razón por la cual en ocasiones se entiende que el parlamento ya no es el soberano es, naturalmente, a causa de la Ley de las Comunidades Europeas de 1972. Esta Ley, de acuerdo con una voz autorizada en la materia:

«... atribuyó, o pretendió atribuir, una posición especial al derecho comunitario dentro del Reino Unido, y más concretamente desde la decisión en el caso Factortame, en el que por primera vez una ley del parlamento fue "inaplicada" por ser contraria al derecho comunitario; cierta diferencia de opinión ha existido sobre si la judicatura británica ha consentido una revolución jurídica abandonando un elemento crucial en la doctrina de la soberanía parlamentaria, ${ }^{47}$

A diferencia de la Ley de las Comunidades Europeas, la Ley de Derechos Humanos de 1998 y la legislación que hizo posible la "devolución" en los territorios no ingleses del Reino Unido, formalmente no suponen un desafío similar al principio de la soberanía del parlamento. Sin embargo, aunque formalmente respeten dicho principio, se puede argumentar que, en la práctica, producen el efecto de limitar el alcance de la soberanía parlamentaria. Lo que resulta indudable es que hoy en día existe mucha menos confianza en la aplicabilidad y relevancia del este principio que cuando Dicey escribió sus reflexiones, y que se ha iniciado el debate sobre si debería haber un catálogo bri-

45 DICEY, Law of the Constitution, págs. 65-66. Hemos modificado ligeramente las palabras textuales de DICEY, ya que él se refirió a la Ley de la Unión (en singular), como si la Unión hubiese quedado asegurada solo por una ley del parlamento inglés, en lugar de exigir el apoyo de los dos parlamentos, el inglés y el escocés.

46 Vid. BOGDANOR, Devolution in the United Kingdom, cap. 3.

47 G. MARSHALL, "The Constitution: Theory and Interpretation", en V. BOGDANOR (Ed.), The Constitution in the Twentieth Century (Oxford University Press, 2003), pág. 50, así como las referencias allí citadas. 
tánico de derechos, un Bill of Rights británico. ${ }^{48}$ Debido a que la soberanía parlamentaria ya no es una doctrina de la constitución de carácter inamovible, hoy puede ser posible promulgar un texto constitucional; y debido a que existe cierto escepticismo respecto al valor de esa doctrina, se han elevado ciertas voces para solicitar que se elabore un texto constitucional. No obstante, una constitución de ese tipo tendría que enfrentarse en un primer momento con el problema de si la Ley de las Comunidades Europeas ha limitado o no la soberanía del parlamento, y de si la limitación a dicha soberanía que en la práctica han supuesto la Ley de Derechos Humanos y la legislación sobre la "devolución" debería recogerse en la constitución. La aprobación y promulgación de un texto constitucional tendría que hacer frente directamente a la doctrina de la soberanía del parlamento.

\section{CONCLUSIONES}

Nos hemos preguntado si la elaboración y promulgación de una constitución británica es factible. Nuestra respuesta es que no existe ningún motivo por el que dicho proyecto no sea factible; no existe ninguna razón por la cual, al menos solo entre los sistemas democráticos, Gran Bretaña no sea capaz de promulgar una constitución. Los problemas que acarrea esa empresa, sin embargo, son formidables. Algunos de esos problemas son similares a los que han tenido que enfrentarse otros países cuando han querido redactar una constitución y que han sido resueltos satisfactoriamente; otros problemas, sin embargo, son más específicos de Gran Bretaña, como ocurre concretamente con los problemas que supone elaborar el texto de una constitución que en parte no ha sido codificada, y con los derivados de la necesidad de hacer frente a la doctrina de la soberanía del parlamento. Lo que resulta indudable es el hecho de que la elaboración de un texto constitucional resulta, al menos en cierta medida, un ejercicio político y jurídico, así como también un ejercicio intelectual; y también que el "es" no puede separarse completamente del "debe ser". La elección de lo que debe incluirse en la constitución inevitablemente implica, como hemos argumentado, decisiones de tipo normativo. Quizás, sin embargo, sea ya el momento de que comencemos a tomar esas decisiones, pues no podremos saber lo que nuestra constitución es en realidad, y mucho menos sabremos comprenderla, hasta que intentemos elaborar y promulgar un texto constitucional.

48 Vid. F. KLUG, "A Bill of Rights: Do We Need One or Do We Already Have One? (2007) P.L. 701-719. Vid. también el "Justice Report, A British Bill of Rights: Informing the Debate", 2007. 
TiTLE: Enacting a British Constitution: Some Problems.

ABSTRACT: It has, from time to time, been suggested that Britain would be better off with a constitution on which the terms are to be found in a print document, known and accessible to all citizens. The purpose of this article is to consider the issue of feasibility, or more precisely, the problems which have to be resolved if it is decided to enact such a British Constitution. The first fundamental problem is to decide what should be included, and the second one is to decide bow should be the constitution elaborated, ratificated and amendet.

RESUMEN: De vez en cuando se apunta que sería mejor que Gran Bretaña contase con una constitución cuyos términos se encontrasen en un documento impreso, conocido y accesible a todos los ciudadanos. La finalidad de este artículo es considerar la cuestión de la factibilidad, o, mejor dicho, los problemas que hay que resolver si se decide promulgar una Constitución Británica. El primer problema fundamental consiste en determinar qué debería incluirse en dicha constitución, y, el segundo, decidir cómo debería elaborarse, ratificarse y modificarse esa constitución.

KeY WorDs: Constitutional reform, Electoral process, Jurisprudence, Political parties. liticos.

Palabras Clave: Reforma constitucional, procedimiento electoral, jurisprudencia, partidos po- 